\title{
Endoplasmic reticulum stress mediates nitric oxide-induced chondrocyte apoptosis
}

\author{
KOJI TAKADA, JUN HIROSE, SOICHIRO YAMABE, YUSHUKE UEHARA and HIROSHI MIZUTA \\ Department of Orthopaedic Surgery, Faculty of Life Sciences, Kumamoto University, Chuo-ku, Kumamoto 860-8556, Japan
}

Received October 10, 2012; Accepted December 24, 2012

DOI: $10.3892 /$ br.2013.52

\begin{abstract}
Nitric oxide (NO) is one of the most important mediators of chondrocyte apoptosis, which is a notable feature of cartilage degeneration. While apoptosis of chondrocytes is induced by p53, NO can also induce endoplasmic reticulum (ER) stress, which may be involved in the process of NO-induced chondrocyte apoptosis. The aims of this study were to determine whether NO-induced ER stress (ERS) leads to apoptosis of chondrocytes and to investigate the temporal relationship between the expression of $\mathrm{C} /$ EBP-homologous protein (CHOP), an ERS-associated apoptotic molecule, and the expression of p53 during apoptosis in NO-stimulated chondrocytes. Rat chondrocytes were stimulated by sodium nitroprusside (SNP), a NO donor. Real-time polymerase chain reaction (PCR) was performed to analyze the mRNA expression of CHOP, glucose-regulated protein (GRP78) and p53. Apoptosis of chondrocytes was quantified using an enzyme-linked immunosorbent assay (ELISA). SNP-treated chondrocytes showed an increase in CHOP and GRP78 mRNA expression and underwent apoptosis. Sodium 4-phenylbutyrate (PBA), an ERS inhibitor, reduced CHOP and GRP78, as well as SNP-stimulated apoptosis of chondrocytes, without affecting the SNP-dependent generation of NO. In addition, the blockade of CHOP following siRNA transfection reduced SNP-induced apoptosis of chondrocytes. The CHOP expression increased after apoptosis was detected in the SNP-treated chondrocytes, whereas the p53 expression increased prior to apoptosis. These data demonstrated that NO-induced ERS leads chondrocytes to apoptosis, although this effect appears to be limited to persistent impairment of NO stimulation. These findings may provide insight into the pathology of cartilage degeneration.
\end{abstract}

Correspondence to: Dr Koji Takada, Department of Orthopaedic Surgery, Faculty of Life Sciences, Kumamoto University, 1-1-1 Honjo, Chuo-ku, Kumamoto 860-8556, Japan

E-mail: ytakada514@yahoo.co.jp

Key words: nitric oxide, endoplasmic reticulum stress, chondrocyte, apoptosis, p53

\section{Introduction}

Nitric oxide (NO) is a significant inducer of chondrocyte apoptosis, a feature of cartilage degeneration in diseases such as osteoarthritis (OA) (1-3). NO-stimulated chondrocytes increased the expression of tumor suppressor p53 via phosphorylation of the p38 mitogen-activated protein kinase, resulting in the enhanced transcription of Bax, a proapoptotic member of the B-cell lymphoma 2 (Bcl-2) family $(4,5)$. Accumulation of Bax in the mitochondria leads to cell apoptosis through the release of cytochrome $c$ from the mitochondria (4). Studies showing that the reduction of the p53 expression inhibits NO-induced apoptosis of chondrocytes provide additional evidence of p53 participation in such apoptosis $(4,5)$.

Endoplasmic reticulum (ER) stress, provoked by an imbalance between the load of unfolded proteins in the ER and the capacity of the ER, leads to the accumulation of unfolded or misfolded proteins in the ER (6). Mammalian cells induce specialized responses to recover or maintain ER function by attenuating general translation, upregulating the expression of ER chaperone proteins, such as the $78 \mathrm{kDa}$ glucose-regulated protein (GRP78), and by activating the endoplasmic reticulum-associated protein degradation system (6). However, if these protective responses fail and ER stress (ERS) persists, specialized apoptotic pathways, such as the enhanced expression of C/EBP-homologous protein (CHOP) and the activation of ER-associated caspase 12, are activated to eliminate the damaged cells (7). Previous studies showed that articular chondrocytes in OA cartilage experience ERS during cartilage degeneration $(8,9)$. ERS induced by pharmacological ERS inducers such as tunicamycin and thapsigargin may induce apoptosis of chondrocytes (10). Previously, we demonstrated that the number of chondrocytes exhibiting ERS correlates with the number of apoptotic chondrocytes in osteoarthritic cartilage (11). Thus, ERS is important in chondrocyte apoptosis during the process of cartilage degeneration.

In their study, Oliver et al (12) showed that $\mathrm{NO}$ is a potent inducer of ERS in chondrocytes. However, NO has not been demonstrated to induce sufficient ERS to induce apoptosis in chondrocytes. In addition, little is known about the correlation between the ERS- and p53-mediated apoptotic pathways induced by NO in chondrocytes.

The objective of this study was to determine whether NO-induced ERS leads to apoptosis in chondrocytes by exam- 
ining whether NO-induced apoptosis in cultured chondrocytes was suppressed by attenuating ERS using the chemical chaperone ERS inhibitor sodium 4-phenylbutyrate (PBA) or by blocking the ERS-associated apoptotic pathway with siRNA against CHOP. In addition, we investigated the temporal relationship between the expression of $\mathrm{CHOP}$ and p53 during chondrocyte apoptosis.

\section{Materials and methods}

Reagents. Sodium nitroprusside (SNP), a NO-donor, was purchased from Sigma (St. Louis, MO, USA). PBA was purchased from Calbiochem (San Diego, CA, USA). Dulbecco's modified Eagle's medium (DMEM) was purchased from Nacalai Tesuque (Kyoto, Japan). Fetal bovine serum (FBS), collagenase type II, and trypsin-ethylenediaminetetraacetic acid (EDTA) were purchased from Invitrogen (Carlsbad, CA, USA).

Chondrocyte isolation and culture. The animal experiments in this study were designed according to the Guidelines for Animal Experimentation of Kumamoto University and were approved by the Animal Experiment Committee of Kumamoto University. Rat articular chondrocytes were isolated from slices of femoral head cartilage from 5-week-old Wistar rats (Japan SLC Inc., Hamamatsu, Japan) by a sequential enzyme digestion method using collagenase type II as described in a previous study (13). Isolated chondrocytes were plated in flasks at a density of $5 \times 10^{4}$ cells $/ \mathrm{cm}^{2}$ in DMEM supplemented with $10 \% \mathrm{FBS}, 100 \mathrm{U} / \mathrm{ml}$ streptomycin and $100 \mathrm{U} / \mathrm{ml}$ penicillin. The culture medium was replaced every 2 days. After 5 days in culture, the cells were detached using trypsin-EDTA and plated on culture plates at a density of $10 \times 10^{4}$ cells $/ \mathrm{cm}^{2}$. Three days after passage, the cells were incubated in serum-free medium with or without PBA ( $3 \mathrm{mM})$ for $12 \mathrm{~h}$ and then treated with $\operatorname{SNP}(0,0.5,1$ or $2 \mathrm{mM})$ for $24 \mathrm{~h}$.

siRNA transfection. Stealth Select RNAi specific for Chop (RSS355093) and Stealth RNAi negative control Hi GC (12935-400) were purchased from Invitrogen. Rat chondrocytes were seeded at $5 \times 10^{4}$ cells $/ \mathrm{cm}^{2}$ in antibiotic-free medium. After $24 \mathrm{~h}$, the cells were transfected with $10 \mathrm{nM}$ siRNA duplexes using lipofectamine RNAiMAX for $36 \mathrm{~h}$ (Invitrogen) according to the manufacturer's instructions. Following transfection, the cells were incubated in serumfree medium for $12 \mathrm{~h}$ and then treated with SNP $(1 \mathrm{mM})$ for $24 \mathrm{~h}$.

Nitrite/nitrate assay. Total NO was measured as its breakdown products, nitrite and nitrate, by the Griess reaction using a nitrate/nitrite colorimetric assay kit (Cayman, Ann Arbor, MI, USA) according to the manufacturer's instructions. Briefly, culture medium from chondrocytes was reacted with nitrate reductase and its cofactor for $1 \mathrm{~h}$ at room temperature. After color development by the addition of Griess reagent, the amount of nitrate/nitrite was determined by absorbance at $540 \mathrm{~nm}$.

Enzyme-linked immunosorbent assay (ELISA) for apoptosis. The extent of cultured apoptosis of chondrocytes was analyzed using Cell Death Detection ELISA ${ }^{\text {PLus }}$ (Roche Applied Science, Mannheim, Germany), according to the manufacturer's instructions. Following treatment, the cell lysate was incubated for $2 \mathrm{~h}$ at room temperature with anti-histone-biotin and anti-DNA-peroxidase antibodies. The absorbance of the samples was measured at 405 and $490 \mathrm{~nm}$. For each experiment, the amount of protein in the cell lysate was assessed in separate wells, using the Quick Start Bradford protein assay (Bio-Rad Laboratories, Richmond, CA, USA) to normalize the extent of cell apoptosis (11). For each experiment, the apoptosis enrichment factor was calculated as the absorbance (A405-A490 nm) of the cells treated with agents/absorbance (A405-A490 nm) of untreated control cells.

RNA extraction and real-time polymerase chain reaction (PCR). Total RNA was extracted from cultured cells using the RNeasy mini kit (Qiagen, Valencia, CA, USA) in combination with DNA digestion using DNase (Qiagen) and reverse-transcribed using the High Capacity RNA-tocDNA kit (Applied Biosystems, Foster, CA, USA). The procedures were performed according to the manufacturer's instructions. Quantitative real-time RT-PCR analysis was performed on an Applied Biosystems 7300/7500 Real-time PCR system using the TaqMan Gene Expression Master mix (Applied Biosystems) and TaqMan Gene Expression assays for Chop (Rn00492098-g1), Grp78 (Rn01435771_g1), p53 (Rn00755717_m1), and Gapdh (Rn99999916_s1) (Applied Biosystems). Reactions were carried out under the conditions: 2 min at $50^{\circ} \mathrm{C}$ and $10 \mathrm{~min}$ at $95^{\circ} \mathrm{C} ; 40$ cycles of $15 \mathrm{sec}$ at $95^{\circ} \mathrm{C}$ and $1 \mathrm{~min}$ at $60^{\circ} \mathrm{C}$. The relative quantification of the target gene to Gapdh was calculated using the $\Delta \Delta \mathrm{Ct}$ method (User Bulletin no. 2, Applied Biosystems).

Statistical analysis. Data were expressed relative to the mean value of cells treated without agents in each experiment. Statistical analysis was carried out using a one-way analysis of variance (ANOVA) with the Scheffe's post hoc tests. $\mathrm{P}<0.05$ was considered to indicate a statistically significant difference.

\section{Results}

SNP induces apoptosis and ERS in chondrocytes. The generation of NO in SNP-treated chondrocytes was confirmed using the Griess reaction (Fig. 1A). Treating chondrocytes with SNP significantly increased apoptosis of chondrocytes at doses of $\geq 1 \mathrm{mM}$ (Fig. 1B). Chop mRNA expression increased in SNP-treated chondrocytes in a dose-dependent manner (Fig. 1C). SNP also increased Grp78 mRNA expression in chondrocytes (Fig. 1D), although this effect was reduced in a dose-dependent manner, and no significant difference was observed in the level of Grp78 expression between $2 \mathrm{mM}$ SNP-treated chondrocytes and the control cells (Fig. 1D). These results suggest that NO induces ERS in chondrocytes. On the basis of these results, $1 \mathrm{mM}$ SNP was used in subsequent experiments.

ERS inhibitor PBA and Chop knockdown reduce SNP-induced apoptosis of chondrocytes. Although PBA had no significant effect on the generation of NO by SNP (Fig. 2A), PBA reduced 

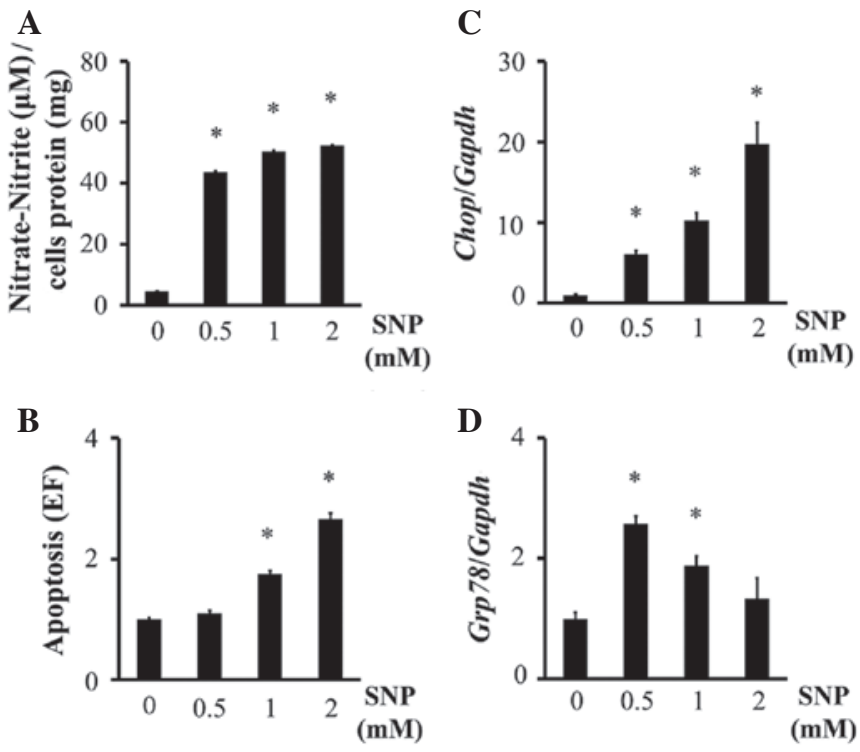

Figure 1. SNP increases ERS-associated mRNA expression and apoptosis in chondrocytes. Chondrocytes were treated with SNP (0-2 mM) for $24 \mathrm{~h}$. (A) NO generation was measured using the Griess reaction. (B) Quantification of apoptosis was performed by ELISA. (C) Chop and (D) Grp78 mRNA expression associated with ERS relative to Gapdh expression was measured by real-time PCR and normalized to samples without SNP. Values are represented as the mean \pm standard error of the mean of three independent experiments performed in triplicate. ${ }^{*} \mathrm{P}<0.05$ vs. samples without SNP. SNP, sodium nitroprusside; NO, Nitric oxide; ERS, endoplasmic reticulum stress.
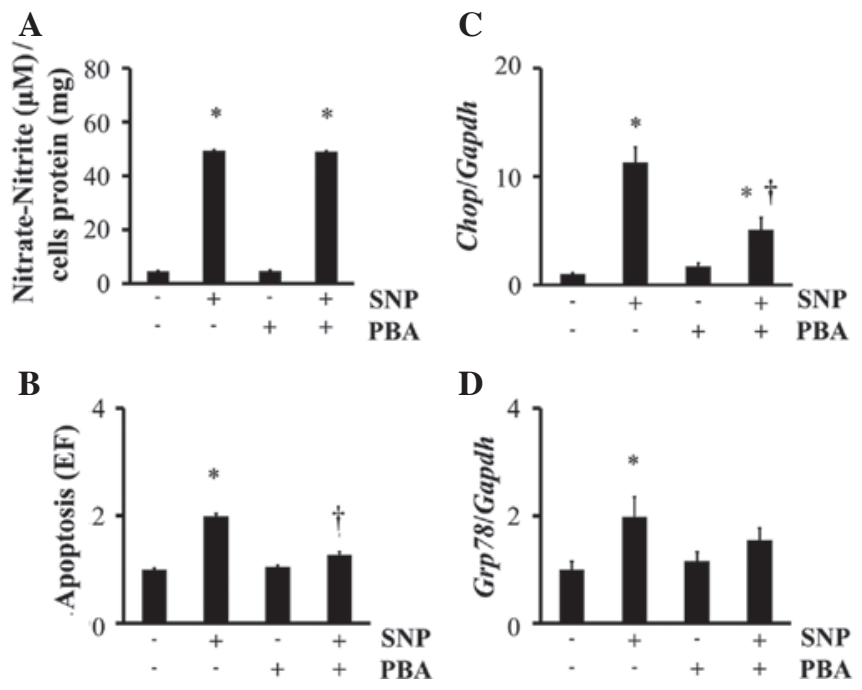

Figure 2. PBA suppresses ERS and apoptosis in chondrocytes. Chondrocytes were pretreated with PBA ( $3 \mathrm{mM}$ ) for $12 \mathrm{~h}$ and then stimulated by SNP (1 mM) for $24 \mathrm{~h}$. (A) NO generation by SNP was measured using the Griess reaction. (B) Chondrocyte apoptosis was analyzed by ELISA. (C) Chop and (D) Grp78 mRNA expression relative to that of Gapdh was measured by real-time PCR. The data were normalized to samples treated without agents. Values are presented as the mean \pm \pm standard error of the mean of three independent experiments performed in triplicate. ${ }^{*} \mathrm{P}<0.05$ vs. samples without agents. ${ }^{\dagger} \mathrm{P}<0.05$ vs. samples with SNP alone. PBA, 4-phenylbutyrate; SNP, sodium nitroprusside; NO, Nitric oxide; ERS, endoplasmic reticulum stress.

apoptosis (Fig. 2B) and the mRNA expression of Chop (Fig. 2C) and Grp78 (Fig. 2D) in SNP-treated chondrocytes.
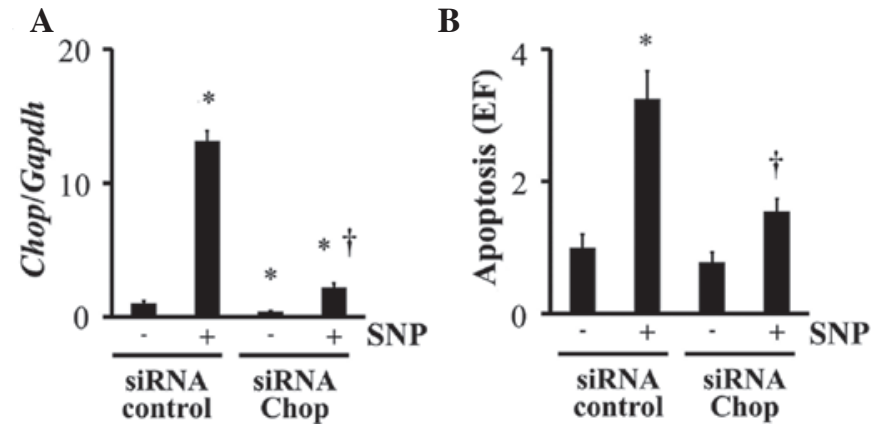

Figure 3. Inhibition of Chop expression reduces SNP-induced chondrocyte apoptosis. Chondrocytes were transfected with Chop or negative control siRNA and then stimulated with SNP (1 mM). (A) Twenty-four hours after stimulation, Chop mRNA expression relative to that of Gapdh was measured by real-time PCR in chondrocytes stimulated with SNP. (B) Chondrocyte apoptosis stimulated by SNP for $24 \mathrm{~h}$ was analyzed by ELISA. Data were normalized to samples treated without agents. Values are presented as the mean \pm standard error of the mean $($ SEM) of three independent experiments performed in triplicate. ${ }^{*} \mathrm{P}<0.05$ vs. samples without agents. ${ }^{\dagger} \mathrm{P}<0.05$ vs. samples treated with negative control siRNA and SNP. SNP, sodium nitroprusside.

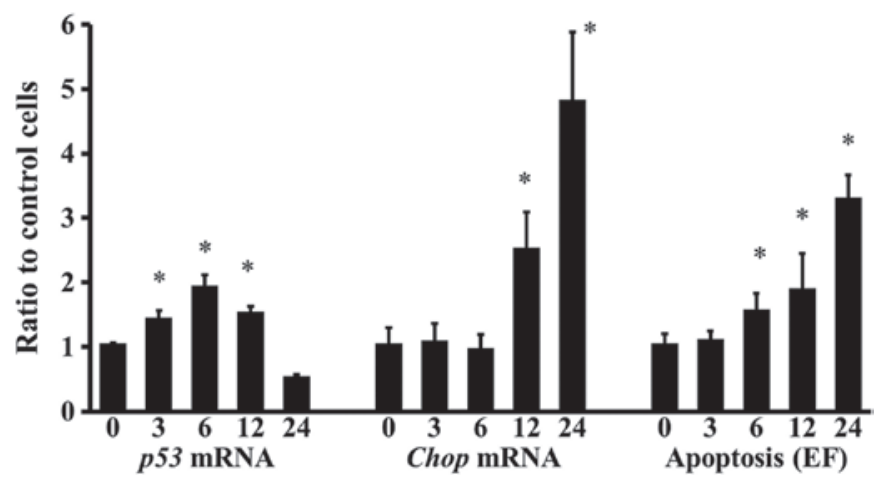

Figure 4. Expression of Chop and p53 and changes in apoptosis in a timedependent manner. Chondrocytes were treated with SNP (1 mM) for $24 \mathrm{~h}$. The mRNA expression of Chop and p53 was measured by real-time PCR. Chondrocyte apoptosis was analyzed by ELISA. Data were expressed relative to the mean value of control cells treated without SNP at each time point. Values of the graph are presented as the mean \pm standard error of the mean of three independent experiments performed in triplicate. ${ }^{*} \mathrm{P}<0.05$ vs. samples prior to treatment. SNP, sodium nitroprusside.

Chop knockdown was performed by siRNA transfection. Chop siRNA significantly inhibited Chop expression in chondrocytes, even when the cells were stimulated with SNP (Fig. 3A). siRNA treatment also reduced SNP-induced apoptosis of chondrocytes (Fig. 3B).

Temporal relationship between the expression of Chop and p53 and the induction of apoptosis. Three hours after chondrocytes were treated with SNP, p53 expression significantly increased, but Chop expression and apoptosis were not enhanced (Fig. 4). p53 expression of chondrocytes achieved a peak $6 \mathrm{~h}$ following treatment and was kept upregulated up to $12 \mathrm{~h}$ (Fig. 4). Chop expression increased in a time-dependent manner $12 \mathrm{~h}$ after SNP stimulation, whereas apoptosis of chondrocytes increased in a time-dependent manner $6 \mathrm{~h}$ after the stimulation (Fig. 4). 


\section{Discussion}

In this study, we observed that SNP induced apoptosis of chondrocytes and SNP-stimulated chondrocytes showed an increase in the CHOP and GRP78 mRNA expression. These results are consistent with those of previous reports using $S$-nitroso- $N$-acetylpenicillamine (SNAP), another NO donor (12). Therefore, NO is an inducer of ERS in chondrocytes. In addition, we found that PBA treatment as well as the blockade of the CHOP expression was able to suppress NO-induced apoptosis of chondrocytes.

PBA has been reported to be a chemical chaperone that reduces the misfolding and mislocalization of mutant $\alpha 1$-antitrypsin (14) and the aggregation of Pael-R (15), and attenuates ERS in liver cells, mouse embryonic fibroblasts (16) and neuronal cells $(15,17)$. In a preliminary experiment (data not shown), PBA was confirmed to also be useful as an ERS inhibitor in chondrocytes, based on the observation that PBA inhibited the expression of GRP78 and CHOP, as well as the tunicamycin-stimulated apoptosis in chondrocytes, a typical ERS inducer. Therefore, the suppressive effect of PBA on NO-stimulated apoptosis in chondrocytes determined that NO-induced ERS led chondrocytes to apoptosis. The results of the CHOP knockdown experiment also supported this conclusion.

In their study, Kim et al $(4,5)$ demonstrated that p53 was responsible for NO-induced apoptosis of chondrocytes. In the present study, the p53 expression in NO-treated chondrocytes increased prior to the induction of apoptosis and decreased with sustained stimulation by $\mathrm{NO}$, despite the increased apoptosis. By contrast, the upregulation of $\mathrm{CHOP}$ was induced following the induction of NO-stimulated apoptosis in chondrocytes. These results suggest that p53 plays a role in the apoptosis of chondrocytes mainly at the acute stage of NO stimulation, although this was not the case for ERS. However, similar to apoptosis, the CHOP expression was found to increase with sustained stimulation. Qu et al (18) demonstrated that ERS prevented p53 stabilization via glycogen synthase kinase- $3 \beta$ and inhibited p53-mediated apoptosis. Taking these findings into consideration, we assumed that ERS initially inhibited p53-mediated NO-induced apoptosis of chondrocytes, but as NO stimulation progressed, the persistent impairment altered the ERS responses of chondrocytes from protective to apoptotic. However, the mechanism of this switch remains unclear. Therefore, additional studies are required to clarify the role of ERS in NO-induced apoptosis.

The limitation of this study is that our observations are based on experiments using exogenous NO generated by SNP, a NO donor, rather than NO synthases-generated endogenous NO. However, endogenous NO may also induce ERS and lead to apoptosis in several cell types, such as pancreatic $\beta$ cells (19) and macrophages (20). Therefore, endogenous NO-induced apoptosis of chondrocytes may also be mediated by ERS.

In conclusion, findings of the present study have demonstrated that ERS contributed to NO-induced apoptosis of chondrocytes, using pharmacological attenuation of ERS and blockade of the ERS-associated apoptotic pathway. However, the contribution of ERS appears to be limited to persistent impairment of NO stimulation. Previous studies have demonstrated that $\mathrm{NO}$ is the molecule most responsible for apoptosis of chondrocytes during cartilage degeneration (1-3). Therefore, our results support that ERS is involved in apoptosis of chondrocytes of degenerated cartilage. The importance of ERS in NO-induced apoptosis may provide insight into the pathology of cartilage degeneration. However, additional studies are required to investigate the role of ERS in cartilage biology.

\section{References}

1. Blanco FJ, Ochs RL, Schwarz H and Lotz M: Chondrocyte apoptosis induced by nitric oxide. Am J Pathol 146: 75-85, 1995.

2. Hashimoto S, Takahashi K, Amiel D, Coutts RD and Lotz M: Chondrocyte apoptosis and nitric oxide production during experimentally induced osteoarthritis. Arthritis Rheum 41: 1266-1274, 1998.

3. Pelletier JP, Jovanovic DV, Lascau-Coman V, Fernandes JC, Manning PT, Connor JR, Currie MG and Martel-Pelletier J: Selective inhibition of inducible nitric oxide synthase reduces progression of experimental osteoarthritis in vivo: possible link with the reduction in chondrocyte apoptosis and caspase 3 level. Arthritis Rheum 43: 1290-1299, 2000.

4. Kim SJ, Hwang SG, Shin DY, Kang SS and Chun JS: p38 kinase regulates nitric oxide-induced apoptosis of articular chondrocytes by accumulating p53 via NFkappa B-dependent transcription and stabilization by serine 15 phosphorylation. J Biol Chem 277: 33501-33508, 2002.

5. Kim SJ, Ju JW, Oh CD, Yoon YM, Song WK, Kim JH, Yoo YJ, Bang OS, Kang SS and Chun JS: ERK-1/2 and p38 kinase oppositely regulate nitric oxide-induced apoptosis of chondrocytes in association with $\mathrm{p} 53$, caspase-3, and differentiation status. J Biol Chem 277: 1332-1339, 2002.

6. Ron D and Walter P: Signal integration in the endoplasmic reticulum unfolded protein response. Nat Rev Mol Cell Biol 8: 519-529, 2007.

7. Gotoh T and Mori M: Nitric oxide and endoplasmic reticulum stress. Arterioscler Thromb Vasc Biol 26: 1439-1446, 2006.

8. Horton WE Jr, Bennion P and Yang L: Cellular, molecular, and matrix changes in cartilage during aging and osteoarthritis. $\mathrm{J}$ Musculoskelet Neuronal Interact 6: 379-381, 2006.

9. Nugent AE, Speicher DM, Gradisar I, McBurney DL, Baraga A, Doane KJ and Horton WE Jr: Advanced osteoarthritis in humans is associated with altered collagen VI expression and upregulation of ER-stress markers Grp78 and bag-1. J Histochem Cytochem 57: 923-931, 2009.

10. Yang L, Carlson SG, McBurney D and Horton WE Jr: Multiple signals induce endoplasmic reticulum stress in both primary and immortalized chondrocytes resulting in loss of differentiation, impaired cell growth, and apoptosis. J Biol Chem 280: 31156-31165, 2005.

11. Takada K, Hirose J, Senba K, Yamabe S, Oike Y, Gotoh T and Mizuta H: Enhanced apoptotic and reduced protective response in chondrocytes following endoplasmic reticulum stress in osteoarthritic cartilage. Int J Exp Pathol 92: 232-242, 2011.

12. Oliver BL, Cronin CG, Zhang-Benoit Y, Goldring MB and Tanzer ML: Divergent stress responses to IL-1beta, nitric oxide, and tunicamycin by chondrocytes. J Cell Physiol 204: 45-50, 2005.

13. Hirose J, Ryan LM and Masuda I: Up-regulated expression of cartilage intermediate-layer protein and ANK in articular hyaline cartilage from patients with calcium pyrophosphate dihydrate crystal deposition disease. Arthritis Rheum 46: 3218-3229, 2002.

14. Burrows JA, Willis LK and Perlmutter DH: Chemical chaperones mediate increased secretion of mutant alpha 1-antitrypsin (alpha 1-AT) Z: a potential pharmacological strategy for prevention of liver injury and emphysema in alpha 1-AT deficiency. Proc Natl Acad Sci USA 97: 1796-1801, 2000.

15. Kubota K, Niinuma Y, Kaneko M, Okuma Y, Sugai M, Omura T, Uesugi M, Uehara T, Hosoi T and Nomura Y: Suppressive effects of 4-phenylbutyrate on the aggregation of Pael receptors and endoplasmic reticulum stress. J Neurochem 97: 1259-1268, 2006. 
16. Ozcan U, Cao Q, Yilmaz E, Lee AH, Iwakoshi NN, Ozdelen E, Tuncman G, Görgün C, Glimcher LH and Hotamisligil GS: Endoplasmic reticulum stress links obesity, insulin action, and type 2 diabetes. Science 306: 457-461, 2004.

17. Qi X, Hosoi T, Okuma Y, Kaneko M and Nomura Y: Sodium 4-phenylbutyrate protects against cerebral ischemic injury. Mol Pharmacol 66: 899-908, 2004.

18. Qu L, Huang S, Baltzis D, Rivas-Estilla AM, Pluquet O, Hatzoglou M, Koumenis C, Taya Y, Yoshimura A and Koromilas AE: Endoplasmic reticulum stress induces p53 cytoplasmic localization and prevents p53-dependent apoptosis by a pathway involving glycogen synthase kinase-3beta. Genes Dev 18: 261-277, 2004.
19. Oyadomari S, Takeda K, Takiguchi M, Gotoh T, Matsumoto M, Wada I, Akira S, Araki E and Mori M: Nitric oxide-induced apoptosis in pancreatic beta cells is mediated by the endoplasmic reticulum stress pathway. Proc Natl Acad Sci USA 98: 10845-10850, 2001.

20. Gotoh T, Oyadomari S, Mori K and Mori M: Nitric oxideinduced apoptosis in RAW 264.7 macrophages is mediated by endoplasmic reticulum stress pathway involving ATF6 and CHOP. J Biol Chem 277: 12343-12350, 2002. 